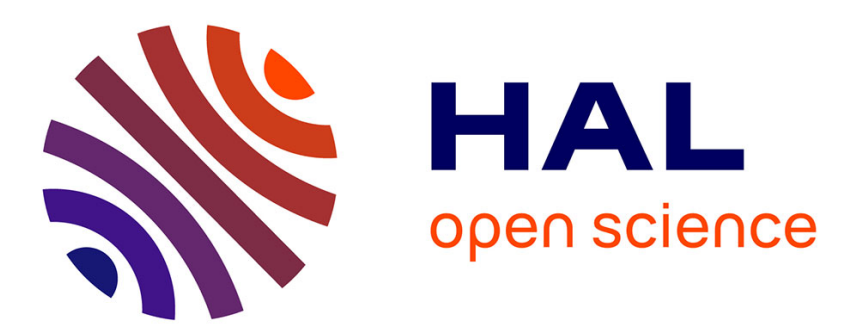

\title{
libmask: Protecting Browser JIT Engines from the Devil in the Constants
}

\author{
Abhinav Jangda, Mohit Mishra, Benoit Baudry
}

\section{To cite this version:}

Abhinav Jangda, Mohit Mishra, Benoit Baudry. libmask: Protecting Browser JIT Engines from the Devil in the Constants. Annual Conference on Privacy, Security and Trust, Dec 2016, Auckland, New Zealand. hal-01382971

\section{HAL Id: hal-01382971 \\ https://inria.hal.science/hal-01382971}

Submitted on 17 Oct 2016

HAL is a multi-disciplinary open access archive for the deposit and dissemination of scientific research documents, whether they are published or not. The documents may come from teaching and research institutions in France or abroad, or from public or private research centers.
L'archive ouverte pluridisciplinaire HAL, est destinée au dépôt et à la diffusion de documents scientifiques de niveau recherche, publiés ou non, émanant des établissements d'enseignement et de recherche français ou étrangers, des laboratoires publics ou privés. 


\title{
libmask: Protecting Browser JIT Engines from the Devil in the Constants
}

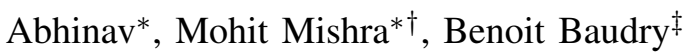 \\ *Indian Institute of Technology (BHU) Varanasi \\ \{abhinav.student.apm11, mohit.mishra.cse11\}@iitbhu.ac.in \\ ${ }^{\dagger}$ BrightEdge Technologies, Inc. \\ mmishra@brightedge.com \\ †INRIA Rennes, France. \\ benoit.baudry@inria.fr
}

\begin{abstract}
JavaScript (JS) engines are virtual machines that execute JavaScript code. These engines find frequent application in web browsers like Google Chrome, Mozilla Firefox, Microsoft Internet Explorer and Apple Safari. Since, the purpose of a JS engine is to produce executable code, it cannot be run in a non-executable environment, and is susceptible to attacks like Just-in-Time (JIT) Spraying, which embed return-oriented programming (ROP) gadgets in arithmetic or logical instructions as immediate offsets. This paper introduces libmask, a JIT compiler extension to prevent the JIT-spraying attacks as an effective alternative to XOR based constant blinding. libmask transforms constants into global variables and marks the memory area for these global variables as read only. Hence, any constant is referred to by a memory address making exploitation of arithmetic and logical instructions more difficult. Further, these memory addresses are randomized to further harden the security. The scheme has been implemented and evaluated as a librddy extension to Google V8 scripting engine with optimizations that contain performance overhead and make libmask a feasible approach. We demonstrate that libmask masks all the constants in JITed code, and effectively raise the bar for JIT-spray and JITROP attacks. The average overhead incurred upon memory is less than 300 kilobytes, while in most benchmarks the memory overhead is less than $10 \mathrm{~KB}$. The average performance overhead observed with optimizations measures is $5.31 \%$. Further, this new approach shows a modest performance improvement over currently deployed constant blinding technique in Google V8.
\end{abstract}

\section{INTRODUCTION}

At the heart of web browsers lie JavaScript engines virtual machines that execute the JavaScript code. Some of the popular JavaScript engines are: Google V8 (in Google Chrome)[30], SpiderMonkey (in Mozilla Firefox)[31], Chakra (in Microsoft IE and Edge)[32], JavaScriptCore (in Apple Safari)[33], and Mozilla's Rhino (completely written in Java)[34]. With such proliferation of web browsers in the market, it becomes equally important to harden them against security vulnerabilities and exploits. Attackers often abuse vulnerabilities and bugs to conduct a successful exploit on the system or application. In response to these exploits, multiple defense mechanisms have been proposed and deployed in modern browsers. Data Execution Prevention (DEP)[4] is widely deployed across browsers and systems. DEP is a technique that prevents input data (from the attacker) from being executed, thus preventing payload injection attacks. However,
Shacham et al. [18] showed that DEP can be bypassed, using a technique called Return-Oriented Programming (ROP)[18]. ROP is an effective code-reuse attack that exploits a set of Turing-complete code snippets called ROP gadgets in the program and chain them together to deploy an attack.

JIT-spraying attacks allow an attacker to bypass data execution prevention (DEP) and memory randomization (ASLR). For this, the attacker embeds a ROP gadget as an immediate offset in XOR instructions. Subsequently, the attacker forces the JIT-compiler to emit many of these malicious instructions so that the attacker's chance of guessing the location of one of those instructions is high.

This paper introduces libmask, a non-XORing constant blinding scheme, to prevent the so-called JIT-spraying attacks. These attacks embed ROP gadgets in arithmetic and/or logical instructions as immediate offsets. The motivation behind this scheme is to reduce the performance overhead and increase security of the currently deployed XOR based constant blinding. For example, in case of Google V8, only integer constant of size greater than 4 bytes are blinded. A constant is first XORed with a random key to generate the obfuscated constant. The constants is XORed back again when the original constant needs to be used. Athanasakis et al. in [17] has shown that XOR constant blinding technique can be bypassed. Their technique contains constants, which are less than 4 bytes and hence, are emitted as such in the JIT code. To mitigate this issue, we replace a constant with a randomized address.

The fundamental idea is to replace each constant with a random address. This random address stores the value of the constant. This is achieved by replacing constants with a global variable in the source code. All constants within the source code are allocated at random addresses by random shuffling and adding random padding. The memory area of these random addresses is then marked as read-only. We then present several optimizations to improve the performance. These optimizations includes Register Allocation and Constant Caching to improve the performance of constant masked code and masking constants in parallel to decrease the JIT compilation overhead.

We evaluated libmask against XOR based constant blinding in Google V8 and discovered that libmask performs better than XOR based constant blinding both in terms of 
security and performance.

a) Contributions: This paper makes the following contributions:

- We introduce the design of libmask, a JIT Compiler extension as an alternative to XOR based constant blinding (Section III).

- We present various optimizations for libmask to improve the performance of JIT code and reduce JIT compiler overhead (Section IV).

- We plugged in libmask into Google V8 and performed extensive evaluations on Octane Benchmarks [16] (Section V). Our evaluations show that:

- The average performance overhead of libmask is $5.31 \%$.

- The average memory requirement of libmask is less than $300 \mathrm{~KB}$, while a number of benchmarks show an average requirement of just $10 \mathrm{~KB}$.

- Iibmask performs better than XOR based constant blinding extensively deployed in modern browsers like Google Chrome.

\section{BACKGROUND}

\section{A. Just-In-Time Compiler}

A Just-in-time (JIT) compiler generates native code during the execution of the program. JIT compilation combines two traditional approaches: ahead of time compilation and interpretation. JIT compilers emit native code during program run-time. Like interpreters, they translate bytecode to machine code continuously but employ caching of translated code to avoid re-translating the previously translated code. Several modern run-time environments employ JIT compilation techniques like most implementations of Java, Microsoft .NET Framework, or Google's V8 Javascript Engine.

However, the JITed code is predictable in nature. In actual practice, extremely minimal variations are introduced in the JITed code: the JIT compiler always emits out the same native code for a certain piece of source code. This predictability nature puts its security under question since adversaries can predict the native code with accuracy.

\section{B. ROP and JIT Spray}

Return-oriented programming is a non code-injection attack technique. It differs from the classical return-to-libc[18] in the way that it utilizes small instruction sequences (ending with ret instruction) present in either the binary or libraries linked to the application. These instruction sequences are called gadgets. The following example illustrates how ROP attack works.

Consider loading a constant into a register, which will allow to save the value in the stack, and enabling it to be popped when required. The following instruction would pop the value from the stack into the EAX register and return the address on the stack's top, which can be the address to the next gadget.

1 pop eax;

2 ret;

\begin{tabular}{|c|}
\hline pop eax; ret; \\
\hline 0x00dead; \\
\hline next gadget's address; \\
\hline
\end{tabular}

Fig. 1. Stack representation of the gadget

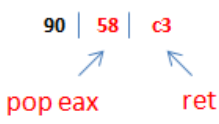

Fig. 2. Intended Gadget - Loading a constant in a register and popping it when required.

The stack diagram in Figure 1 explains how the above gadget helps to return to the next gadget, which further can be chained to perform a successful exploit.

JIT Spray[5], a form of ROP attack exploits a JIT Compiler's behavior and bypasses Address Space Layout Randomization [18] and DEP [4]. As mentioned earlier, JIT compilers are predictable in the way that the JITed code shows minimal variations across multiple compilations. Also, a JIT compiler generates executable code, and hence it must run in an executable environment. Therefore, a JIT compiler is usually exempt from DEP. A JIT Spray attack works by performing heap spraying[36] with the generated native code that facilitates arbitrary code execution. In case of web browsers, heap spraying makes use of the JavaScript code and spray the heap by creating large strings, followed by making copies of the long string with a shellcode. These copies are stored in an array, till the point where sufficient memory has been sprayed. This ensures that the exploit would work.

\section{Constant Blinding/Masking}

There are two ways a constant in the source code could land up in the executable code: (i) as a value stored by the compiler close to the code (without executing it as code), or (ii) as an immediate instruction operand.

For the first case, it suffices to add padding, e.g. NOP insertion, randomizing the address location of the value, making it hard to guess for the adversaries. For the later case, one of the currently deployed defenses in modern browsers effective against JIT Spray attacks is constant blinding. This involves a generated random key (or cookie) used to XOR an immediate operand, and then XORed again when it is actually used. For instance, assume the following sample JavaScript code:

$1 \operatorname{var} a=0 \times 9058 \mathrm{C} 3$

The native code (without any constant blinding mechanism) would include the constant as it is. Note that this constant can potentially be exploited since, it can act as an intended gadget.

Figure 2 shows that the combination of 58 and $c 3$ can act as gadget, which can pop a value as and when required from the stack. To prevent this to happen, modern web browsers deploy constant blinding, wherein the constant is XORed with a random key (called cookie) and XORed again when the constant needs to be used. This way, the constant never ends up as it is in the JIT buffer, raising the bar for the adversary 


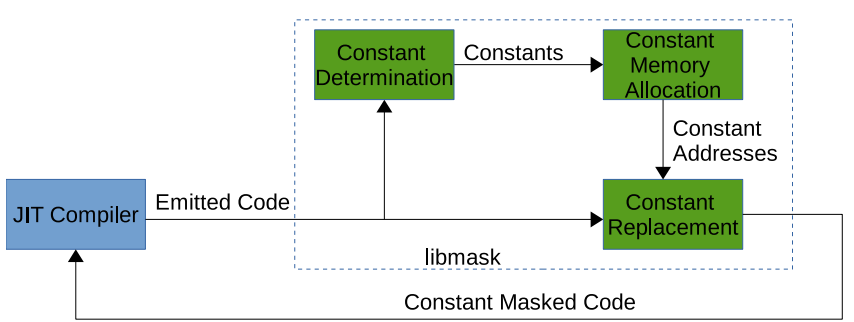

Fig. 3. Design and working of libmask

to predict the value without knowing the cookie's value. The variable $a$ is never showed up as $0 \times 9058 \mathrm{c} 3$, but as $0 \times 826 \mathrm{c} 95$ with cookie's value used being $0 \times 123456$. Now, when variable $b$ wants to use the original value of variable $a, a$ is XORed again with the same cookie in its operation.

$1 \mathrm{a}=0 \times 9058 \mathrm{c} 3 \wedge 0 \times 123456 / /=826 \mathrm{c} 95$

$2 \ldots$

$3 \mathrm{~b}=\mathrm{a} \wedge 0 \times 123456$

The machine code generated to implement constant blinding for above example is given below:

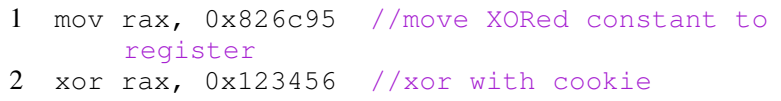

As can be observed from above, it becomes hard to guess the original constant's value, since that is never showed up in the native code as it is. However, there are two operations required to obfuscate and use the value of the constant. The current scheme takes care of integer constants of size more than 4 bytes only (e.g. in Google V8). Gadgets can be formed from integers of less than 4 bytes also [17].

\section{DESIGN}

libmask performs constant masking in the code generated by a JIT compiler. It reads the code emitted by JIT compiler, disassembles it, performs constant masking, assembles the constant-masked code (CMC), and returns the CMC to the JIT compiler. This CMC is then executed by the virtual machine. Figure 3 shows the design and working of libmask. Currently, libmask supports x86-64 architecture, and hence our discussion will stick around with x86-64 architecture in this paper.

Constant masking via libmask is divided into three phases: (i) Constant Determination, (ii) Constant Memory Allocation, and (iii) Constant Replacement. Constant Determination phase determines all the constants in the machine code. Constant Memory Allocation allocates the memory for the constants (constant memory) and marks it as read-only after shuffling and padding. Constant Replacement phase replaces each constant found in the machine code with it's respective address in constant memory.

\section{A. Constant Determination}

After disassembling the code generated by JIT compiler, this phase traverses each machine instruction inside the code. If any one of the operands to a machine instruction is a constant, then the constant and the position of the machine instruction are recorded.

\section{B. Constant Memory Allocation}

After traversing the machine code, the set of all constants is shuffled. Further, random padding is added after each constant. This ensures that the constants are allocated at randomized addresses. After allocating memory, the storage slot corresponding to each constant is initialized with the value of the constant. Finally, this constant memory (memory holding up the constants) area is marked as read-only.

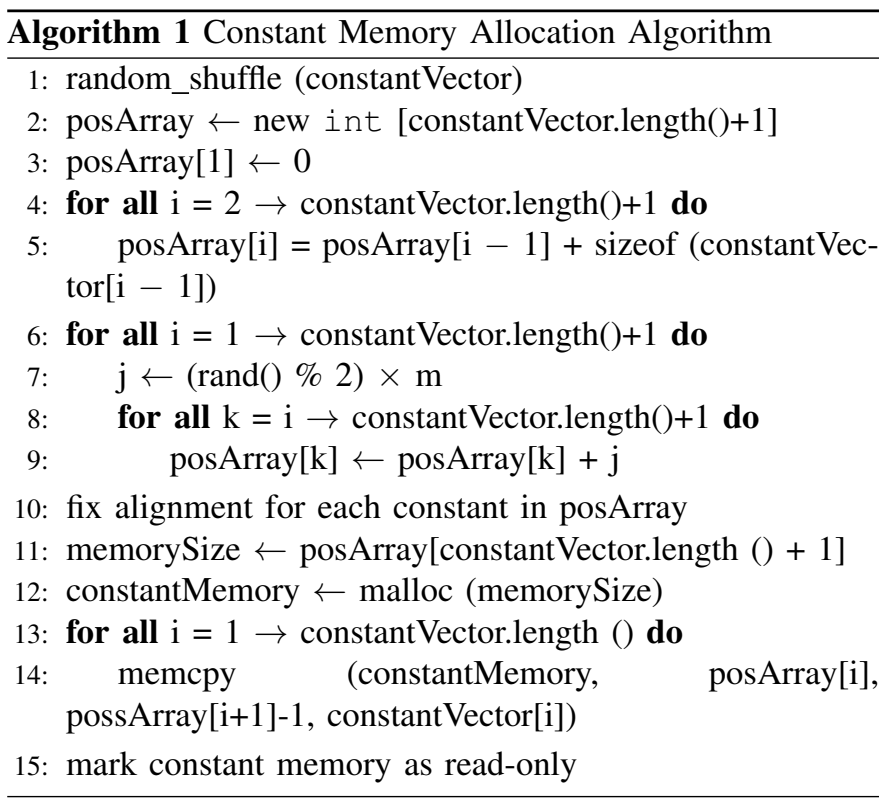

Algorithm 1 presents constant memory allocation algorithm. It takes two arguments: (i) constantVector, which is a vector of constants as an argument and (ii) m, which is the length of each padding. Line 1 randomly shuffles constantVector. Line 2 creates posArray, which contains the position (in bytes) in constant memory for each constant. Lines 3 - 5 set the initial position for each constant based on the size of the previous constant in the array. Lines 6 9 add a random padding of atmost $m$ bytes after every constant. Line 10 adds the required alignment for every constant and updates the posArray. Lines 10 - 11 allocate the constant memory, initialize the memory locations with the constant, and mark the constant memory as read-only.

\section{Constant Replacement}

After Constant Memory Generation phase, Constant Replacement will replace constant in machine instruction with its address in the constant memory. In x86-64 architecture, one of the operand in instructions can also be an address. For example, the add instruction, can take a constant, register, or a memory location as one of its operand. This memory location will be first de-referenced by the CPU to obtain the operand value. This phase rewrites the instruction in the machine code with the address of constant in constant memory.

\section{Example}

In this section, we present an example of the working of our technique. For this example we assume an x86 32-bit 


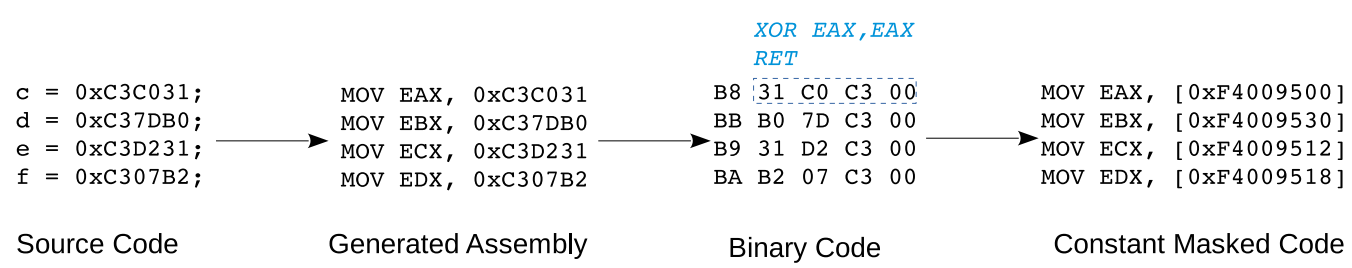

Fig. 4. Example

architecture for simplicity and understanding. Figure 4 shows an example of constant masking using libmask in the source code. The source code contains five constants. These constants are the gadgets required for calling mprotect [17]. The JITed contains these constants, and hence these constants can be exposed by an attacker. On the other hand, the constant masked code generated by libmask replaces these constants with random addresses. These random addresses are accessed by the CPU to obtain the constant. The random addresses are different for every execution of the source program.

\section{Optimizations}

libmask presented in Section III could result in high performance overhead as given in Section V. This performance overhead is due to the following reasons:

1) Low performance of CMC: An immediate value in the original machine code is replaced by an address to produce the CMC. Since, a memory access is a costly operation compared to using an immediate operand, CMC suffers from this bottleneck.

2) Time taken in diversification of code: The whole process of diversification takes time proportional to the number of constants to be blinded. In Section V, MandreelLatency benchmark suffers the highest performance overhead. MandreelLatency benchmarks measures the JIT compiler latency.

To decrease the performance overhead due to the above mentioned issues, the following optimizations are performed.

\section{A. Parallel Division of the Machine Code}

Optimizations presented in Section IV-B and Section IV-C require the JITed code to be divided into loop-free subsequences. A loop-free subsequence is defined as a machine code instruction sequence, which does not contain a loop i.e. a loop-free subsequence does not contain a backward branch. Every backward branch divides the code into two loop-free subsequences: (i) one between the previous loopfree subsequence and the target of the backward branch, (ii) and the other between the target of the backward branch and the backward branch itself.

In this section, we present a parallel algorithm for dividing a machine code into loop-free subsequences. To divide the machine code into loop-free subsequences in parallel, different threads are created at first. The number of threads is set to be equal to the number of CPU cores. The machine code is then divided equally to each of the threads. Each thread then examines each instruction individually. If an instruction is a backward branch, then the target of the backward branch is recorded into the thread's local database. Finally, each thread's local database is merged serially to the main thread database.

\section{B. Register Allocation}

Main Memory access is a costly operation as compared to access from register or using immediate operands. Accessing same memory address more than once is costlier than accessing the memory address once, storing the value in a register, and accessing that register when required. Therefore, we developed our Register Allocation optimization algorithm to decrease the memory access overhead.

Initially, the constant masked code is divided into loopfree subsequences as given in Section IV-A. In a loop-free subsequence, for each register $r$ all parts of a loop-free subsequence between each access of $r$ is determined. Let us call each of these parts of a loop-free subsequence as access range for a register $r$. Hence, in each of the access range of register $r$, there are no accesses to the $r$ i.e. for each instruction in access range of $r, r$ is neither used as a source operand or a destination operand. In each of the access range for a register $r$, the occurrence of each constant memory address as an operand to any instruction is counted. In an access range of register $r$, for a constant memory address with maximum number of accesses, push $r$ and mov $r$, [<constant memory address $>$ ] instructions are inserted before the first instruction of access range for $r$, each occurrence of the constant memory address is replaced by $r$, and pop $r$ instruction is inserted after the last instruction of address range of $r$. This process is then done for all access range of all registers. Note that push, pop, and mov instructions do not change the flag register.

If the access range for a register $r$ is the loop, then above technique also has the advantage of bringing the push and mov instructions outside of the loop.

\section{Constant Caching}

Since, constants have to be loaded from the constants memory, the CPU can take advantage of the memory locality. To improve the locality provided by the technique in Section III, we develop Constant Caching optimization.

In a loop-free subsequence, if one constant is accessed then the CPU can take the advantage of the memory locality, to also load other constants of a loop-free subsequence in the cache. To acheive this, instead of shuffling all constants in the constant un-masked code, Constant Caching optimization 
first shuffles the constants of a loop-free subsequence and then shuffles each group of constants of a loop-free subsequence.

Alogrithm 2 represents the Constant Caching Algorithm. Lines 1 - 2 shuffle constants for each loop-free subsequence. Line 3 shuffles each loop-free subsequence. To add Constant Caching in our technique, Line 1 of Algorithm 1 is replaced by Alogrithm 2 .

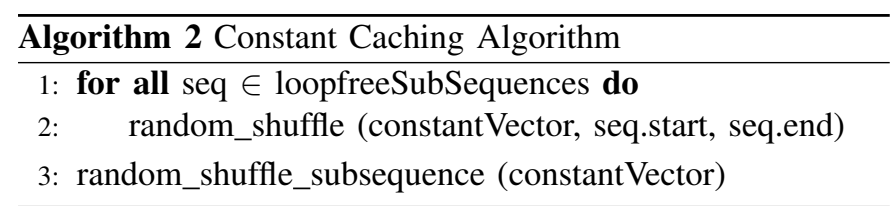

\section{Parallel Constant Determination}

The Constant Determination phase given in III-A is serial. Hence, Constant Determination phase would perform poorly on benchmarks with huge number of constants. To solve this issue, we developed Parallel Constant Determination optimization. In this optimization, the disassembled code generated by the JIT compiler is divided equally to different threads. The number of threads is set equal to the number of CPU cores. Each thread then examines each instruction. If the operand to any instruction is a constant, it is recorded in a threads local database. Each thread's local database is then merged serially to the main thread database.

\section{Evaluation}

In this section, we provide our implementation of libmask, evaluation techniques, environment, and results. Our evaluation is based on the performance, memory requirement, and security impact.

\section{A. Implementation}

Google Chrome occupies over 60\% [15] of the market share of web browsers. Hence, it was imperative to implement and evaluate our technique on Google V8. Like most virtual machines, Google V8 has a Garbage Collector and a JustIn-Time compiler. However, V8 does not use any byte code representation. Instead, it directly converts JavaScript code to machine code.

\section{B. Environment}

We evaluated our implementation on a 4 core $2.30 \mathrm{GHz}$ Intel Core i7-3610QM machine with 6 GB of RAM running Fedora 21 with Linux Kernel version 3.19 and executing Octane 2.0 JavaScript Benchmarks [16].

\section{Performance Evaluation}

We performed performance evaluation on different configurations. In each of these configurations, we fixed $\mathrm{m}=8$, which is the length of each padding in Algorithm 1. The configurations are:

- V8 configuration is Google V8 without libmask and XOR based constant blinding disabled.
- Iibmask configuration is Google V8 with libmask presented in Section III without any optimization.

- Iibmask $+O$ configuration is Google V8 integrated with libmask with all optimizations enabled.

- $X O R$ is Google V8 with XOR based constant blinding enabled. Google V8 implements a XOR based Constant Blinding by XORing an integer of size more than 4 bytes with a random key and then XORing it again when it is actually used.

- Iibmask $+O+4 b$ configuration is Google V8 integrated with libmask with all optimizations enabled but only constants of greater than 4 bytes are masked. This configuration is used to compare with $X O R$ configuration.

Each benchmark was executed five times for each of the configurations and average time was calculated. Figure 5 shows the performance overhead for libmask over $V 8$, libmask $+O$ over $V 8$, and $X O R$ over libmask $+O+4 \mathrm{~b}$. Observe that with the optimizations enabled the overhead of libmask has significantly decreased. MandreelLatency benchmark, which measures compiler latency shows significant performance overhead of $50.75 \%$ with libmask configuration. This is because Mandreel benchmark consists of over 19,000 integer constants and blinding all of them incurs noticeable overhead. However, with optimizations enabled libmask, this overhead reduces to $19 \%$ because the constants are now being masked in parallel. In Mandreel benchmark which shows the highest overhead, there are large number of constants in the functions with high amount of hotness. On the other hand, for the benchmarks with low overhead like Richards the number of constants in the functions with high amount of hotness are very less. The average performance overhead observed with optimizations enabled is $5.31 \%$. Figure 5 also shows that $X O R$ configuration has some performance overhead over $1 i$ bmas $k+O+4 b$. This performance overhead can be attributed to the following reasons:

- XOR based constant blinding adds 2 instructions in the machine code, while 1 ibmask $+O+4 b$ adds only 1 instruction.

- Memory addressing is a costly operation but with Constant Caching and Register Allocation optimizations, instead of memory access either register access or cache access is used to obtain the constant.

- XOR based constant blinding does not implement techniques to decrease the JIT compilation latency. With libmask working over the source code in parallel, there is a significant decrease in the overhead.

\section{Memory Requirements}

Table I shows the number of integer constants and size of constant memory in KBs for each benchmark. The size of constant memory is directly proportional to the number of constants. Hence, Mandreel benchmark with 274842 constants requires $3 \mathrm{MB}$ for constant memory and zlib with lowest number of constant requires only $0.7 \mathrm{~KB}$ for constant memory. On an average, the size of constant memory required is only $295.7 \mathrm{KBs}$. 


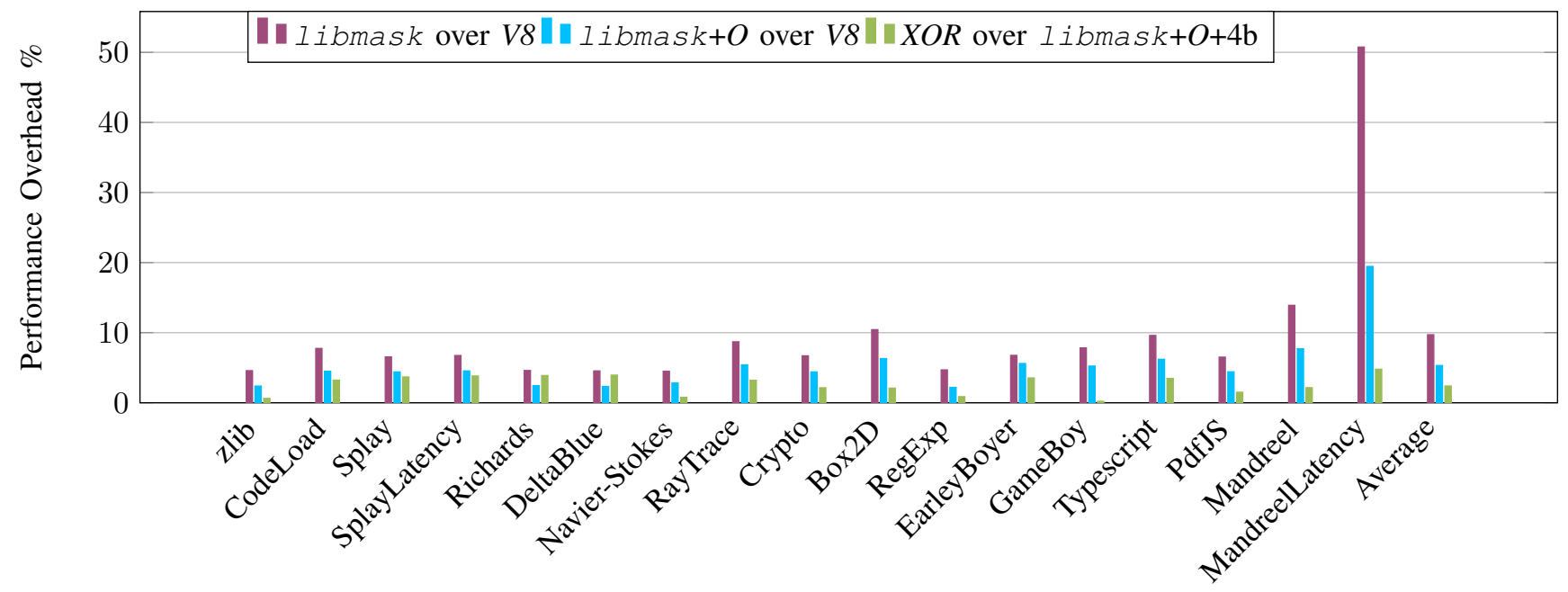

Fig. 5. Performance Overhead (in \%) for different configurations

\begin{tabular}{|c|c|c|}
\hline Benchmark & $\begin{array}{l}\text { \# of Integer } \\
\text { Constants }\end{array}$ & $\begin{array}{l}\text { Constant Memory } \\
\text { Size (in KBs) }\end{array}$ \\
\hline Richards & 98 & 1.1 \\
\hline DeltaBlue & 115 & 1.3 \\
\hline Crypto & 800 & 9.6 \\
\hline RayTrace & 176 & 2.1 \\
\hline EarleyBoyer & 555 & 6.6 \\
\hline Splay & 98 & 1.1 \\
\hline GameBoy & 6211 & 74.5 \\
\hline CodeLoad & 72 & 0.86 \\
\hline Mandreel & 274842 & 3298.1 \\
\hline zlib & 59 & 0.7 \\
\hline Typescript & 4276 & 51.3 \\
\hline PdfJS & 80446 & 965.3 \\
\hline RegExp & 312 & 3.7 \\
\hline Navier-Stokes & 199 & 2.3 \\
\hline Box2D & 1475 & 17.7 \\
\hline Average & 24648 & 295.7 \\
\hline
\end{tabular}

Number of Integer Constants And Constant Memory Size (IN KBS) FOR OCTANE BENCHMARKS

\section{E. Security Evaluation}

We performed security evaluation of libmask using two approaches: (i) security analysis and (ii) implementing real world attacks.

1) Security Analysis: The security provided by libmask is based on the fact that constants appearing in the source code are replaced by random memory addresses. Hence, the attacker is not able to exploit the original constants any longer, since, they completely get masked under randomized addresses and are not available in the JITed code. Moreover, in each run of the program the constants are masked to different addresses, i.e. no constant gets the same memory address across different runs of the program, thereby significantly reducing any chance for attacker to guess the memory address. Since, these are random addresses, it is highly improbable that the attacker could get a useful gadget.

Assuming there are $n$ constants in the source program, there are $n$ ! ways to arrange constants in the constant memory and there are $2^{n}$ different ways to add padding in the constant memory. Hence, for $\mathrm{n}$ constants there are $2^{n} \times n$ ! different addresses. Table II depicts the number of possible array layouts for different values of $n$. With $n=32$, there are $1.1 \times 10^{45}$ possible memory addresses. For $\mathrm{n}=16$, there are $1.3 \times 10^{18}$ possible memory addresses. Therefore, the probability of guessing the correct array layout is far too low to consider to be feasible. For $n=16$, the probability is $7.7 \times 10^{-19}$.

We chose two attacks presented in [17] for evaluating the security provided by libmask. One JavaScript attack is shown in Figure 6. This attack contains 10 distinct constants, hence there are $2^{10} \times 10 !=3715891200$ possible addresses, which can mask the constants. The second JavaScript attack is shown in Figure 7. This attack contains 7 distinct constants, hence, there are $2^{7} \times 7 !=645120$ possible addresses, which can mask the constants. We executed both these attacks on V8 with libmask and all the optimizations enabled. We found that constants did not appear as such in the instructions, instead they were masked by random addresses. We further found that everytime we execute the attacks on V8 with libmask and all the optimizations enabled, the disassembly of these random addresses is different from what is required to produce the attack. No gadget chaining could be then performed to allow arbitrary code execution.

\section{RELATED WORK}

A lot of work has been done to develop defenses against code re-use attacks. One of the first ideas for raising the bar of attack by randomization was presented in [1]. Several ways of achieving this like substituting equivalent instructions, instruction reordering, extra jump or call insertions and many others were introduced by Cohen [1]. These ideas were further enhanced in [2].

Code Randomization has been one of the most common defense against code re-use attacks. Randomization techniques for achieving code randomization includes instruction and 


\begin{tabular}{|r|r|r|r|}
\hline Number of Constants (n) & Cases for inserting padding $\left(2^{n}\right)$ & Constant Arrangments (n!) & Possible Memory Addresses (2 $\left.{ }^{n} \times n !\right)$ \\
\hline 2 & $2^{2}$ & $2 !$ & 8 \\
\hline 4 & $2^{4}$ & $4 !$ & 384 \\
\hline 8 & $2^{8}$ & $16 !$ & $10^{7}$ \\
\hline 16 & $2^{16}$ & $32 !$ & $1.3 \times 10^{18}$ \\
\hline 32 & $2^{32}$ & $1.1 \times 10^{45}$ \\
\hline
\end{tabular}

Possible Constant MEMORy AdDRESSES FOR DIFFERENT NUMBER OF CONSTANTS

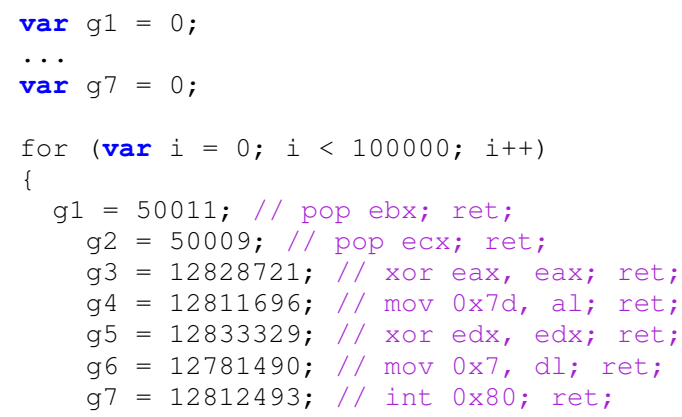

Fig. 6. Attack 1

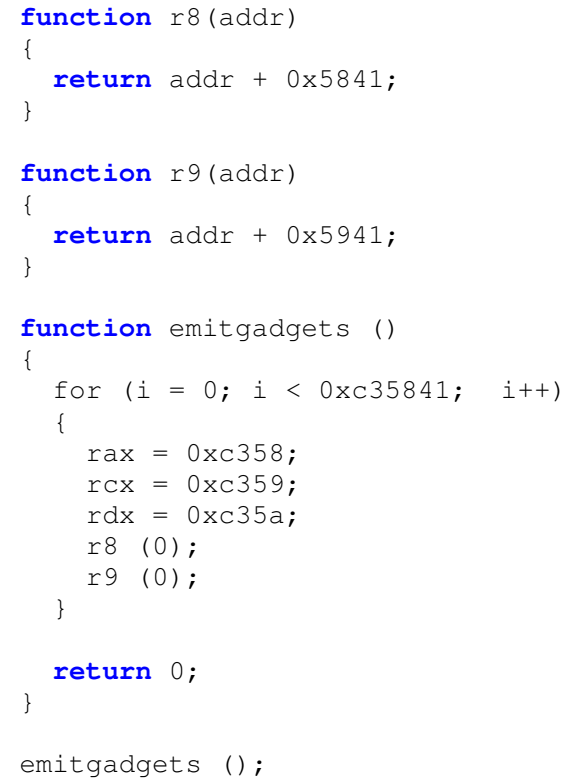

Fig. 7. Attack 2

basic block reordering, register re-allocation, instruction substitution and NOP insertion. These techniques are implemented by [3], [6], [7] in compiler. [9], [8] and [29] implements these techniques in virtual machines and JIT. [10], [11] uses static binary re-writing to implement above techniques. [8] applies above techniques on dynamically generated code. These implementations show that diversification has a positive impact on software security with negligible performance overhead. [8] relies on static NOP insertion techniques while [29] has shown that even less performance overhead could be achieved using dynamic adaptive NOP insertion.
One of the most common defenses to achieve constant blinding is to XOR the constant with a randomly generated key (cookie) and XOR the obtained value again with the key whenever constants are used. We compared our approach with this technique and found that our approach is significantly better than this approach both in security and in performance. The Chakra JS engine used in Internet Explorer as its JIT engine employs several defenses like NOP insertion and XOR based Constant Blinding. [17] has shown that even if JIT uses the above approach as defense an attacker can still successfully attack the system by generating some specific gadgets.

Song et al. showed in [12] that JIT buffers can be exploited with the help of code injection techniques if the buffer is both writable and executable or even temporarily writable at times. A multi-threaded generated code is more prone to above attack because the switch between writable and executable leaves a time window for the application of exploit. To avoid such exploits they also proposes a dynamic code generation architecture which utilizes a separate process and shared memory.

Address Space Layout Randomization (ASLR) is an operating system level randomization technique. It places significant areas of the program like stack and memory obtained from mmap at random addresses. This technique is used by all major operating systems (Windows, Linux and Mac OS). Although this technique has several benefits it is not a sufficient security measure against code reuse attacks. [18] has shown that ASLR has very low entropy and it would take just a few minutes to find the base address. DEP [4] and SafeSEH [28] are another widely adopted lightweight protection mechanism. However, like ASLR these too can be bypassed using techniques like return-to-libc and ROP-based exploits.

Bubble [37] is an effective technique to mitigate heap spraying wherein diversity is introduced at random locations in the heap. Inserting special interrupting values in strings at random locations in the heap at the time when a string is stored in memory breaks the attackers' assumption of memory homogeneity, thereby preventing heap spraying attack.

JITDefender [13] is one way of improving JIT compiler security. It hinders JIT spraying attacks by making all HLL code pages as non-executable. These pages are changed to executable by the compiler once the control enters in the generated code and are changed back on return to the compiler on runtime. This prevents an attacker from redirecting any other branch to dynamically generated code. INSeRT [22] add code randomization through a white box approach, requiring manual changes in the code generation phase of compiler.

Control Flow Integrity was first proposed by Abadi et al.[14]. This technique marks the valid targets of indirect 
control transfers (i.e. function entry points and landing points for returns) with unique identifiers(IDs) and then inserts IDchecks before each indirect call or return instruction. They then identify the set of valid targets through a precise control flow graph construction and enforcing control flow only to this set for each indirect transfer instruction. This technique was improved by Zhang et al in [23].

DynamoRIO [24], Valgrind [25], PIN [27] and Strata [26] are binary rewriting tools. These tools are used for increasing security, profiling, instrumentation and debugging.

\section{CONCLUSION AND Future WORK}

In this contribution, we presented libmask, a non-XOR based Constant Masking technique for protecting browser JIT engines against JIT spraying attacks that leverage constants. libmask is capable of masking constants of all sizes, while incurring a modest overhead over performance. libmask replaces constant in the machine code with randomized addresses. We also presented several optimizations for decreasing the performance overhead.

We integrated libmask with Google Chrome's V8 JavaScript Engine. We evaluated the performance and security impact of our technique by executing Octane Benchmarks and compared it with existing mechanism, i.e. XOR based constant blinding. We found that our approach masks all constants in the JITed code and the average performance overhead we obtained was $5.31 \%$. We also found out, our approach not only performs better than XOR based constant blinding but also has better security impact than XOR based constant blinding.

As a part of our future work, we intend to further enhance the performance of libmask and explore the possibility of developing hybrid mechanisms presented in academia and industries to harden security against JIT-ROP attacks.

\section{REFERENCES}

[1] F. Cohen. Operating system protection through program evolution. Computers and Security, 12(6):565-584, Oct. 1993.

[2] S. Forrest, A. Somayaji, and D. Ackley. Building diverse computer systems. In Proceedings of the 6th Workshop on Hot Topics in Operating Systems, HotOS '97, pages 67-72, 1997.

[3] C. Giuffrida, A. Kuijsten, and A. S. Tanenbaum. Enhanced operating system security through efficient and fine-grained address space randomization. In Proceedings of the 21st USENIX Security Symposium, pages 475-490, 2012.

[4] S. Andersen and V. Abella, "Data Execution Prevention: Changes to Functionality in Microsoft Windows XP Service Pack 2, Part 3: Memory Protection Technologies," http://technet.microsoft.com/enus/library/bb457155.aspx, 2004.

[5] Dion Blazakis. Interpreter Exploitation: Pointer Inference and JIT Spraying. http://www.semantiscope.com/research/BHDC2010/BHDC2010-Paper.pdf

[6] T. Jackson, B. Salamat, A. Homescu, K. Manivannan, G. Wagner, A. Gal, S. Brunthaler, C. Wimmer, and M. Franz. Compiler-generated software diversity. In S. Jajodia, A. K. Ghosh, V. Swarup, C. Wang, and X. S. Wang, editors, Moving Target Defense, volume 54 of Advances in Information Security, pages 77-98. Springer New York, 2011.

[7] A. Homescu, S. Neisius, P. Larsen, S. Brunthaler, and M. Franz. Profileguided automated software diversity. In Proceedings of the 2013 International Symposium on Code Generation and Optimization, CGO '13, 2013.

[8] A. Homescu, S. Brunthaler, P. Larsen, and M. Franz. librando: Transparent Code Randomization for Just-in-Time Compilers In ACM Conference on Computer and Communications Security, CCS' 13.

[9] J. Hiser, A. Nguyen-Tuong, M. Co, M. Hall, and J. W. Davidson. ILR Where'd my gadgets go? In Proceedings of the 33rd IEEE Symposium on Security and Privacy, S\&P '12, pages 571-585, 2012.
[10] V. Pappas, M. Polychronakis, and A. D. Keromytis. Smashing the gadgets: Hindering return-oriented programming using in-place code randomization. In Proceedings of the 33rd IEEE Symposium on Security and Privacy, S\&P '12, pages 601-615, 2012.

[11] R. Wartell, V. Mohan, K. W. Hamlen, and Z. Lin. Binary stirring: Selfrandomizing instruction addresses of legacy x 86 binary code. In Proceedings of the 19th ACM Conference on Computer and Communications Security, CCS '12, pages 157-168, 2012.

[12] C. Song, C. Zhang, T. Wang, W. Lee, and D. Melski, Exploiting and protecting dynamic code generation, in Proceedings of the 2015 Network and Distributed System Security (NDSS) Symposium, February 2015.

[13] P. Chen, Y. Fang, B. Mao, and L. Xie. JITDefender: A defense against JIT spraying attacks. In Proceedings of the 26th IFIP TC 11 International Information Security Conference, SEC '11, pages 142-153, 2011.

[14] M. Abadi, M. Budiu, U. Erlingsson, and J. Ligatti, Control-flow integrity, in ACM Conference on Computer and Communications Security (CCS), 2005.

[15] Browser Statistics and Trends. http://www.w3schools.com/browsers/browsers_stats.asp [16] Octane 2.0 JavaScript Benchmark. http://octanebenchmark.googlecode.com/svn/latest/index.html

[17] M. Athanasakis, E. Athanasopoulos, M. Polychronakis, G. Portokalidis, S. Ioannidis, The Devil is in the Constants: Bypassing Defenses in Browser JIT Engines in Network and Data Security Symposium, NDSS' 15.

[18] H. Shacham, M. Page, B. Pfaff, E. Goh, N. Modadugu, and D. Boneh. On the effectiveness of address-space randomization. In Proceedings of the 11th ACM Conference on Computer and Communications Security, CCS '04, pages 298-307, 2004.

[19] J. Salwan. ROPgadget tool, 2012. URL http://shellstorm.org/project/ROPgadget/.

[20] V8 Constant Blinding SafePush https://github.com/v8/v8/blob/master/src/ia32/macroassembler-ia32.cc\#L607

[21] V8 Constant Blinding Sa feMove https://github.com/v8/v8/blob/master/src/ia32/macroassembler-ia32.cc\#L597

[22] T. Wei, T. Wang, L. Duan, and J. Luo. INSeRT: Protect dynamic code generation against spraying. In Proceedings of the 2011 International Conference on Information Science and Technology, ICIST '11, pages 323-328, 2011

[23] Practical control flow integrity and randomization for binary executables. In IEEE Symposium on Security and Privacy, S\&P '13, pages 559 - 573, 2013

[24] D. Bruening, T. Garnett, and S. Amarasinghe. An infrastructure for adaptive dynamic optimization. In Proceedings of the 2003 International Symposium on Code Generation and Optimisation, CGO '03, 2003.

[25] N. Nethercote and J. Seward. Valgrind: A program supervision framework. Electronic Notes in Theoretical Computer Science, 2003.

[26] K. Scott, N. Kumar, S. Velusamy, B. R. Childers, J. W. Davidson, and M. L. Soffa. Retargetable and reconfigurable software dynamic translation. In Proceedings of the 2003 International Symposium on Code Generation and Optimisation, CGO '03, 2003.

[27] C.-K. Luk, R. Cohn, R. Muth, H. Patil, A. Klauser, G. Lowney, S. Wallace, V. J. Reddi, and K. Hazelwood. Pin: building customized program analysis tools with dynamic instrumentation. In Proceedings of the ACM PLDI '05, pages 190-200, 2005.

[28] Microsoft Visual Studio 2005, Image has safe exception handlers, http://msdn.microsoft.com/en-us/library/9a89h429\%28v=vs.80\%29.aspx.

[29] A. Jangda, M. Mishra, and B. De Sutter. adaptive: Adaptive Just-In-Time Code Diversification. In Proceedings of MTD, ACM CCS'15, Denver CO, USA, Oct. 2015

[30] https://developers.google.com/v8/?hl=en

[31] URL https://developer.mozilla.org/en-US/docs/Mozilla/Projects/SpiderMonkey

[32] URL https://github.com/Microsoft/ChakraCore

[33] URL http://trac.webkit.org/wiki/JavaScriptCore

[34] URL https://developer.mozilla.org/en-US/docs/Mozilla/Projects/Rhino

[35] Aleph One. Smashing the Stack for Fun and Profit. Phrack 49. URL http://insecure.org/stf/smashstack.html

[36] skypher.com. Heap spraying (2007), http://skypher.com/wiki/index.php

[37] F. Gadaleta , Y. Younan , W. Joosen. BuBBle: A Javascript Engine Level Countermeasure against Heap-spraying Attacks. Proc. 2nd Int'1 Symp. Eng. Secure Software and Systems, 2010.

[38] V8 Runtime Functions https://github.com/v8/v8/blob/master/src/runtime/runtimescopes.cc 